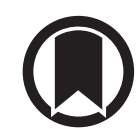

CrossMark

\section{Adults with cystic fibrosis and pulmonologists: new training needed to recruit future specialists}

\author{
To the Editor:
}

The epidemiology and natural history of cystic fibrosis (CF) is changing. Predicted median survival has increased over the past five decades, from less than 12 to more than 45 years in most Western countries, along with an estimated increase of the number of patients: by $20 \%$ in children and by $75 \%$ in adults, over the period 2010 to 2025 [1]. The number of adults (age $\geqslant 18$ years) with CF is already larger than the number of children [2,3]. This is a consequence of improvements in drug therapies and in the management of patients delivered by multidisciplinary teams in specialised CF centres, resulting in prolonged survival; this will be even more pronounced in the future, thanks to the introduction of new CFTR modulators acting on the dysfunctional CFTR protein in epithelial and other cells.

The recent European Respiratory Society (ERS)/European Cystic Fibrosis Society (ECFS) report on the care of adults with CF underlines that lung disease in CF drives most clinical care requirements [4]. Routine ambulatory care is focused on prevention of chronic infection and exacerbations and maintenance of forced expiratory volume in $1 \mathrm{~s}$ and quality of life. This is achieved by long term antimicrobial and mucoactive therapies. When exacerbations occur, these require urgent new antibiotic interventions to mitigate further lung injury. Adults with $\mathrm{CF}$ also face major complications, from life-threatening respiratory infections, which are often due to antimicrobial resistant organisms such as nontuberculous mycobacteria, non-fermenting Gram-negative bacteria, fungi and viruses, to respiratory failure, lung transplantation and end-of-life management. All of these healthcare needs require expertise from trained respiratory physicians. Therefore, the ERS/ECFS task force strongly recommended that pulmonologists who have had specific training in CF should lead the multidisciplinary teams [4].

In a recent systematic review on gaps in the evidence for treatment decisions in CF the authors highlighted that most of the evidence gaps are in the respiratory area; however, the word "adult(s)" is cited four times only [5]. Rошвотнам et al. [6] recently proposed the 10 top research priorities in CF, based on the James Lind Alliance methodology; none of these are specific for adults.

The new challenges include microbiology, with a very high prevalence of multidrug resistant pathogens, the use of disease modifying modulator drug in adults, including the role of these drugs in transplanted patients, age-related complications (e.g. CF-related diabetes, anxiety-depression, osteoporosis, gastrointestinal cancer, haemoptysis, pneumothorax, atherosclerosis and cardiovascular events), solid organ transplantation, mainly lung transplantation, prognostic scores and end-of-life management. Well-being and mental health is also a critical consideration in dealing with changes and transition related to age and disease progression [7].

New ways to deliver care to meet the needs of an older and healthier population are also important. Digital technologies are already in use to replace and augment routine and urgent hospital visits and home self-monitoring may provide an opportunity to improve decision-making around treatment options using machine learning tools. Research on innovation in these areas should be led through well-organised and accredited CF centres with the appropriate infrastructure for translational research.

Advances in care delivery and new antibiotics, mucoactive drugs, correctors and potentiators are reshaping the treatment of CF. It is crucial that the respiratory specialists keep pace with these changes. Many of the

@ERSpublications

Pulmonologists should take the lead in the management of adults with cystic fibrosis. Considerable efforts in research and education are required to ensure all patients to receive a high standard of care. http://ow.ly/cTCb30n22bF

Cite this article as: Blasi F, Elborn JS, Palange P. Adults with cystic fibrosis and pulmonologists: new training needed to recruit future specialists. Eur Respir J 2019; 53: 1802209 [https://doi.org/10.1183/ 13993003.02209-2018]. 


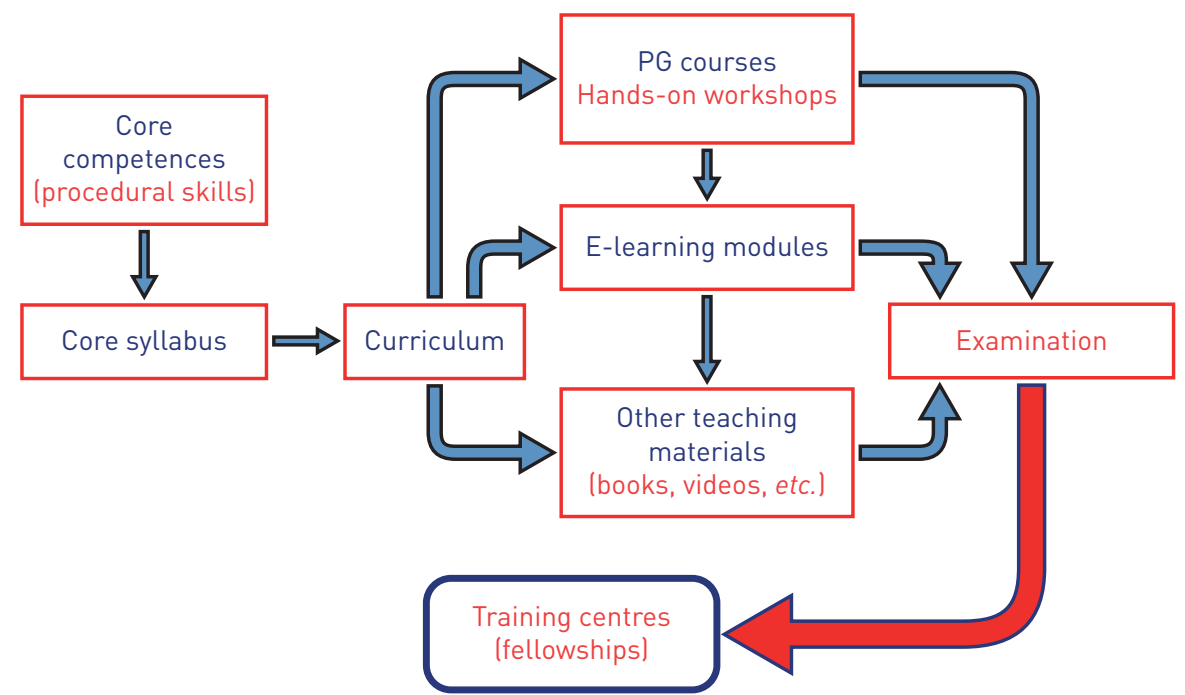

FIGURE 1 Flow chart for a training programme of cystic fibrosis pulmonologists. PG: postgraduate.

drugs used are expensive and some have significant drug/drug interactions and adverse effects. Considerable expertise is required to ensure all patients receive a high standard of care, with optimised drug and physical treatments, and appropriate and holistic integration and management of the care of $\mathrm{CF}$ disease in other organ systems.

These challenges generate new training needs and require renewed efforts in education and training to recruit and retain the next generation of CF specialists.

CF physicians providing care for adults with CF require a range of areas of expertise and should provide holistic care in the context of a multidisciplinary CF team. In addition to their expertise in pulmonology, they should also develop working shared management plans with other specialists to ensure optimal management of CF disease in other organ systems and complications in CF. They should be competent in all aspects of care, including mental health and well-being, and have agreed criteria for input from other specialist teams. This should be the starting point of a training programme designed to prepare individuals who wish to spend a significant proportion of his/her career in the care of adults with CF.

Here we advocate the reconsideration of appropriate training and recruitment to improve disease management in adults with CF. We clearly need a new core curriculum for CF pulmonologists that outlines the training required to deliver optimal specialist care in adults with CF. Specific training centres in countries should be accredited as places that are appropriate for adult CF specialist training. Criteria including the type and volume of the clinical conditions encountered, staffing levels, supervision levels, library and other study facilities and the educational qualifications and experience of teachers and trainers, assessors and supervisory staff are defined according to the European Cystic Fibrosis Society Standards of Care [8].

We propose to urgently prepare and implement strategies to supplement pulmonologists' general knowledge and skills tailored not only to disease burden, but also to health-related and non-health-related management of adults with CF (figure 1).

A concrete sharing of aims, actions, and strategies among the involved stakeholders (scientific societies, patients, healthcare providers, institutions and the pharmaceutical industry) will be needed to ensure we have trained specialist pulmonologists in CF care to deliver optimal care for this life-long disease.

Francesco Blasi ${ }^{1,2}$, J. Stuart Elborn ${ }^{3,4}$ and Paolo Palange ${ }^{5,6}$

${ }^{1}$ Dept of Pathophysiology and Transplantation, Università degli Studi di Milano, Milan, Italy. ${ }^{2}$ Internal Medicine Dept, Respiratory Unit, Adult Cystic Fibrosis Center IRCCS Fondazione Cà Granda Ospedale Maggiore Policlinico, Milan, Italy. ${ }^{3}$ Imperial College and Royal Brompton Hospital, London, UK. ${ }^{4}$ Queen's University Belfast, Belfast, UK. ${ }^{5}$ Dept of Public Health and Infectious Diseases, Sapienza University of Rome, Rome, Italy. ${ }^{6}$ Internal Medicine and Infectious Diseases Dept, Respiratory Unit and Adult Cystic Fibrosis Center, Lorrillard-Spencer Foundation, Policlinico Umberto I Hospital, Rome, Italy.

Correspondence: Francesco Blasi, Dept of Pathophysiology and Transplantation, University of Milano; Internal Medicine Department, Respiratory Unit and Adult Cystic Fibrosis Center, Fondazione IRCCS Ca' Granda Ospedale Maggiore Policlinico, Milan, Italy. E-mail: francesco.blasi@unimi.it 
Received: Nov 202018 | Accepted after revision: Nov 292018

Conflict of interest: F. Blasi reports grants and personal fees from AstraZeneca, Bayer and Pfizer, personal fees from Chiesi, GSK, Guidotti, Insmed, Menarini, Novartis and Zambon, outside the submitted work. J.S. Elborn reports grants from ERS/ECFS, during the conduct of the study. P. Palange has nothing to disclose.

\section{References}

1 Burgel PR, Bellis G, Olesen HV, et al. ERS/ECFS task force on the provision of care for adults with cystic fibrosis in Europe. Future trends in cystic fibrosis demography in 34 European countries. Eur Respir J 2015; 46: 133-141.

2 Simmonds NJ. Ageing in cystic fibrosis and long-term survival. Paediatr Respir Rev 2013; 14: Suppl. 1, 6-9.

3 Parkins MD, Parkins VM, Rendall JC, et al. Changing epidemiology and clinical issues arising in an ageing cystic fibrosis population. Ther Adv Respir Dis 2011; 5: 105-119.

4 Elborn JS, Bell SC, Madge SL, et al. Report of the European Respiratory Society/European Cystic Fibrosis Society task force on the care of adults with cystic fibrosis. Eur Respir J 2016; 47: 420-428.

5 Rowbotham NJ, Smith S, Prayle AP, et al. Gaps in the evidence for treatment decisions in cystic fibrosis: a systematic review. Thorax 2018; in press [https://doi.org/10.1136/thoraxjnl-2017-21085].

6 Rowbotham NJ, Smith S, Leighton PA, et al. These top 10 research priorities in cystic fibrosis developed by a partnership between people with CF and healthcare providers. Thorax 2018; 73: 388-3907.

7 Quittner AL, Saez-Flores E, Barton JD. The psychological burden of cystic fibrosis. Curr Opin Pulm Med 2016; 22: 187-189.

8 Conway S, Balfour-Lynn IM, De Rijcke K, et al. European Cystic Fibrosis Society Standards of Care: Framework for the Cystic Fibrosis Centre. J Cyst Fibros 2014; 13: Suppl. 1, s3-s22. 